\title{
Desarrollo de un enfoque pedagógico crítico en la formación inicial del profesorado para romper con la evaluación tradicional en Educación Infantil
}

Development of a critical pedagogical approach in pre-service teacher training to break with the traditional assessment in Kindergarten

\section{在教师初级培训中打破幼儿教育中传统评估的一种批判性教育 学方法发展}

Разработка критического подхода в начальной педагогической подготовке для того, чтобы порвать с традиционной оценкой в воспитании детей младшего возраста

Raúl A. Barba-Martín

Universidad de Valladolid

raulalberto.barba@uva.es

https://orcid.org/0000-0003-0071-687X

Fechas - Dates

Recibido: 2020-02-22

Aceptado: 2020-05-17

Publicado: 2020-06-31
Cómo citar este trabajo · How to Cite this Paper

Barba-Martín, R. A. (2020). Desarrollo de un enfoque pedagógico crítico en la formación inicial del profesorado para romper con la evaluación tradicional en Educación Infantil. Publicaciones, 50(1), 207-227. doi:10.30827/publicaciones.v50i1.15975 


\title{
Resumen
}

El objetivo del estudio es analizar la transformación de los procesos de evaluación de un grupo de maestras de Educación Infantil y la relación con los modelos formativos vividos. El estudio se enmarca bajo una metodología de investigación-acción participativa, mediante la cual las maestras participaron en un proyecto de formación permanente sobre educación inclusiva. Los resultados muestran cómo el modelo formativo técnico vivido por las maestras durante su formación inicial incidió en una consideración y un uso tradicional de la evaluación; mientras que la formación del proyecto, basada en procesos de autorreflexión, les ayudó a transformar sus concepciones y usos de la evaluación hacia posturas democráticas. La investigación concluye en la necesidad de que la formación inicial del profesorado aúne la vivencia de la evaluación democrática con el desarrollo de un enfoque pedagógico en los estudiantes.

Palabras clave: Evaluación democrática; evaluación participativa; pedagogía crítica; pensamiento crítico

\begin{abstract}
The aim of the study is to analyze the relationship between the transformation of the assessment processes of a group of kindergarten teachers and the relationship with the training models they lived. The study is based under a participatory action-research methodology, through the quality of the teachers participated in an in-service teacher training project on inclusive education. The results shows how the technical teacher training model experienced by the teachers during their pre-service teacher training influenced a traditional consideration and use of the assessment, while the training of the project, based on self-reflection processes, transformed their conceptions and uses of the assessment towards democratic positions. The research concludes in the need for pre-service teacher training to combine the experience of democratic evaluation with the development of a pedagogical approach in students.
\end{abstract}

Keywords: Democratic assessment; shared assessment; critical pedagogy; critical thinking

\section{概要}

本研究的目的是分析对一组幼儿教师的评估过程的转变以及其与现有培训模式的关系。 研究采用了研究-参与性行动的方法, 期间教师参加了关于全纳教育的继续培训项目。研究 结果表明, 在初级培训期间现有对教师的技术培训模式如何影响评估的传统使用与考量; 与此同时, 基于自我反思过程的培训项目帮助其对概念和评估的使用向民主角度转变。研 究得出, 教师初级培训应与从教育学角度促进学生发展的民主评估经验相结合。

关键词: 民主教育; 参与性评估; 批判性教育; 批判性思维

\section{Аннотация}

Цель исследования - проанализировать трансформацию процессов оценки группы педагогов, работающих в области дошкольного образования, и взаимосвязь с учебными моделями, которые они испытали. Исследование проводится в рамках методологии активного участия и исследования, с помощью которой учителя участвовали в проекте непрерывного образования по инклюзивному образованию. Результаты показывают, каким образом техническая модель обучения, используемая преподава- 
телями в ходе их первоначальной подготовки, повлияла на традиционное рассмотрение и использование оценки; в то время как обучение в рамках проекта, основанное на процессах самоанализа, помогло им трансформировать свои концепции и использование оценки в сторону демократических позиций. В исследовании делается вывод о необходимости начальной подготовки учителей, которая должна сочетать опыт демократического оценивания с разработкой педагогического подхода к учащимся.

\section{La evaluación participativa, la evaluación democrática y su conexión con la evaluación en Educación Infantil}

A día de hoy es innegable la gran importancia de la evaluación en relación a la implicación del alumnado en su aprendizaje para mejorarlo. Al menos, así lo abalan la multitud de publicaciones, donde se puede comprobar cómo los modelos de evaluación compartidos, participativos y democráticos suponen un beneficio para los estudiantes en la adquisición de saberes y valores (Boud \& Falchikov, 2007; Dochy, Segers, \& Sluijsmans, 1999; Hortigüela \& Pérez-Pueyo, 2016; López-Pastor, Castejón, Sicilia-Camacho, Navarro-Adelantado, \& Webb, 2011). Estas publicaciones responden al creciente interés de la comunidad educativa por la puesta en práctica de modelos alternativos de evaluación, el cual comprobamos en el ámbito nacional con publicaciones como las de López-Pastor y Pérez-Pueyo (2017). Estos autores, con un libro recopilatorio de buenas prácticas de evaluación en todos los niveles educativos, han mostrado una fuerte tendencia por parte del profesorado en la consideración e implementación de prácticas de evaluación participativas en las escuelas y universidades. Dichas prácticas de evaluación innovadoras son un paso para romper con la evaluación tradicional asentada aún en las escuelas y universidades, cuyo uso se restringe como método de calificación (López-Pastor \& Palacios, 2012).

La participación del alumnado se puede fomentar a través de la implicación en su propia evaluación, pero también en la de sus compañeros y del profesorado. Estas formas implican tres estrategias de evaluación que colocan al alumnado en el centro del proceso: la autoevaluación, la evaluación entre pares o iguales y la evaluación compartida. Estas estrategias han sido estudiadas por numerosos autores, quienes han demostrado sus beneficios en relación a aspectos como el aumento de conciencia del alumnado sobre su aprendizaje y la mayor comprensión de los contenidos (Kennedy \& Allan, 2009), la adquisición de autonomía, individual y grupal (Manrique, López-Pastor, Monjas, \& Real, 2010), la posibilidad de acometer prácticas de aprendizaje autorregulado (Rodríguez, Ibarra, \& Gómez, 2011), la motivación (Andrade \& Du, 2007) o la mejora de las habilidades de reflexión crítica (Fitzpatrick, 2006). Sin embargo, es importante comprender que no son prácticas positivas por sí mismas, sino que es necesario un análisis crítico de las mismas, tal y como afirma en su estudio Beackley (2000). Y es que a estas formas de evaluación innovadoras les acompaña un componente ético inherente, al basarse en el establecimiento de relaciones y la posibilidad de fomentar una participación en diferentes grados. Así, en relación al tratamiento crítico de estos aspectos en la evaluación nos encontramos con la evaluación democrática.

El modelo de evaluación democrática fue acuñado por McDonald (1976). Basado en la propuesta de este autor, la evaluación democrática defiende el uso de la evalua- 
ción más allá de lo técnico, otorgando especial interés a las dimensiones psicológicas, políticas y éticas que subyacen a la evaluación (Santos Guerra, 1993, 2003). En este sentido, las investigaciones sobre evaluación democrática abordan el análisis profundo del uso y aprendizaje de este modelo de evaluación junto a sus valores éticos y pedagógicos intrínsecos. Así podemos encontrar investigaciones cuyas finalidades son resaltar y reflexionar en torno a aspectos tan importantes en los procesos de evaluación democráticos como el empoderamiento del alumnado (Tan, 2008), la crítica a las relaciones entre quien enseña y aprende (Fernández-Balboa, 2005, 2007), los valores de justicia social (Murillo \& Hidalgo, 2015) o su posicionamiento dentro de una pedagogía crítica (Lorente \& Kirk, 2013, 2014). En esta línea, se comprende cómo este modelo de evaluación sigue los principios pedagógicos críticos de autores como Freire, McLaren, Kincheloe o Giroux, quienes consideraban necesario el cuestionamiento por parte del profesorado de todas las ideas y valores subyacentes a sus prácticas y modelos educativos desde un posicionamiento ético, para poder avanzar en la construcción de prácticas, modelos, aulas, escuelas y sociedades realmente democráticas (Freire, 1968/2007; Giroux, 2006; Kincheloe, 2012; McLaren, 2005).

El estudio que se desarrolla a continuación se asienta en la etapa educativa de Educación Infantil, la cual presenta una serie de características particulares en relación a las ideas extraídas hasta el momento sobre la evaluación. De acuerdo con la actual legislación en Educación Infantil (Real Decreto 1630/2006), la observación directa y sistemática debe ser la técnica principal del proceso de evaluación en esta etapa educativa. Las razones para ello vienen apoyadas por su potencial para la detección y comprensión de los procesos de aprendizaje y el desarrollo de habilidades del alumnado en sus primeros años, tales como el aprendizaje de la lectura (Huang, Hsu, Su, \& Liu, 2014) o la motricidad (Herrero-Nivela, Escolano-Pérez, Anguera, \& Acero-Ferrero, 2014). Sin embargo, es necesario comprender que la observación sistemática es una técnica de evaluación centrada en la figura del docente; por ello, en lo últimos años nos encontramos con propuestas de evaluación en la etapa de Educación Infantil dirigidas a complementar la observación sistemática mediante técnicas y prácticas de evaluación participativas (García-Herránz \& López-Pastor, 2015; Páramo \& Castro, 2017; Lopez-Benavente, Urena-Ortin, \& Lopez, 2019). Por tanto, para esta etapa educativa en concreto ya no estamos únicamente ante la necesidad de una reflexión crítica para romper con modelos de evaluación tradiciones, sino también para cuestionar si los existentes, a pesar de ser potenciadores del aprendizaje, son suficientes y democráticos.

Basándonos en estas ideas, el objetivo de nuestro estudio es analizar la transformación de los procesos de evaluación de un grupo de maestras de Educación Infantil y la relación con los modelos formativos vividos, incidiendo en las bases pedagógicas que unen ambos elementos.

\section{Metodología}

Nuestro estudio se ha desarrollado bajo el prisma de la investigación cualitativa. Esta decisión vino fundamentada por la capacidad de esta metodología para interpretar y profundizar en las historias de los participantes a través de sus experiencias, sus acontecimientos y el significado que ellas les otorgan (Denzin \& Lincon, 2012), pudiendo capturar la fluidez y el alcance de esas interacciones (Guzmán-Valenzuela, 2014). 
El método utilizado fue la investigación-acción participativa. El estudio se diseñó en torno a este método al ser el modelo formativo que las maestras estaban desarrollando en el Proyecto en el que se enmarca la investigación y del cual nosotros éramos participantes activos. La investigación-acción participativa desarrolló en las participantes una serie de vivencias, reflexiones, relaciones y conocimientos que se dirigieron a la transformación de sus prácticas educativas y sus contextos escolares y sociales (Greenwood \& Levin, 2007; Kemmis, McTaggart, \& Nixon, 2015). De esta forma, realizar la investigación desde este método nos permitió apoyar dicho proceso de transformación de las maestras al reflexionar, discutir y narrar de forma abierta, fluida y colectiva junto a ellas todo lo acontecido a los cambios en su evaluación de manera continua (Kemmis, 2011), pudiendo así incidir conjuntamente en los momentos clave, las relaciones subyacentes, los factores motivantes de dicha transformación, y su impacto social.

\section{Contexto y participantes}

La investigación se ha realizado en el marco de un Proyecto de Innovación Docente de la Universidad de Valladolid destinado a la formación del profesorado en educación inclusiva a través de investigación-acción participativa (en adelante PID-FPEIAP). El Proyecto se ha basado en el análisis de los contextos escolares de las participantes a través de la autorreflexión crítica y compartida. El grupo se ha reunido cada mes para reflexionar conjuntamente sobre lo acontecido en los contextos durante ese periodo y asentar nuevas concepciones que les permitiesen ir avanzando en la transformación de su práctica educativa, sus aulas, sus escuelas y sus comunidades.

El Proyecto lleva activo desde el curso académico 2013/2014 y la investigación que se presenta en este estudio se realizó durante los tres primeros cursos del Proyecto. En el primer curso académico de andadura del PID-FPEIAP se vincularon una veintena de maestras de tres centros educativos. En cursos posteriores, el número de maestras participantes fue aumentando progresivamente, llegando a alcanzar cincuenta participantes de catorce colegios durante su tercer curso. Todos ellos impartían su docencia en la etapa de Educación Infantil y eran en su mayoría mujeres, por ello cuando nos refiramos específicamente a este colectivo lo haremos en término femenino.

No se realizó ningún tipo de acotación de las participantes para la investigación. Se recogieron datos de todas las maestras participantes y, únicamente, se realizó un muestreo al usar algunos instrumentos, lo cual desarrollamos a continuación.

\section{Diseño y procedimiento de la investigación}

La investigación puede dividirse en tres fases, que se corresponden con cada uno de los cursos académicos sobre los que se tomaron datos y se vivenció el PID-FPEIAP junto a las maestras.

$1^{\text {a }}$ fase: Primeros pasos del Proyecto, delimitación del enfoque de estudio, recogida de los primeros datos y establecimiento de una relación con los participantes. El primer curso académico en el que el PID-FPEIAP se puso en marcha supuso una toma de contacto entre los participantes y con el propio Proyecto. La investigación se diseñó de forma abierta y amplia, cuyo enfoque tenía la finalidad de comprender el PID-FPEIAP desde la voz de las maestras participantes, incidiendo en los principales aspectos destaca- 
dos por ellas, para así poder ser un complemento en sus reflexiones y procesos de transformación. En este primer curso académico la investigación se desarrolló como un estudio exploratorio. Primero se trató de reflexionar en torno a las circunstancias que habían hecho a las maestras participar en el Proyecto y, posteriormente, se recogieron los primeros datos sobre la puesta en marcha. Ser participantes activos en el PID-FPEIAP nos permitió establecer una relación de confianza con las docentes participantes, lo cual resultó beneficioso para la construcción de la investigación.

$2^{\text {a }}$ fase: Asentamiento del Proyecto, profundización en los datos y acceso de nuevos participantes. Las maestras comenzaron el segundo curso del PID-FPEIAP con la idea de asentar las propuestas del año anterior y avanzar en la misma línea con otras nuevas. La investigación se organizó este segundo año como una prolongación del anterior para profundizar en el desarrollo formativo de las maestras. Sin embargo, debido a la participación de un gran número de nuevas maestras dividimos el estudio en dos apartados, conectados a su vez por el enfoque general: por un lado, la realización de un estudio parecido al de la primera fase con las nuevas participantes y, por otro lado, una investigación de profundización para las antiguas participantes.

$3^{\text {a }}$ fase: Reestructuración del PID-FPEIAP y recogida de los últimos datos. Durante este curso académico se produjo el fallecimiento del coordinador del Proyecto, lo cual supuso la detención del PID-FPEIAP y la investigación durante cuatro meses, entre noviembre y febrero. Cuando el Proyecto retoma su andadura, potenciado por el interés de las maestras, juntos consideramos necesario cerrar un ciclo de toma de datos, que hiciesen referencia al periodo del primer coordinador y su forma de organizar la investigación-acción participativa junto a las maestras. De esta manera reestructuramos la investigación para hacer una última recogida de datos antes de terminar el curso académico. Para ello realizamos un análisis de todos los datos obtenidos hasta el momento, extrajimos los temas principales por los que habían navegado los intereses de las maestras y construimos diferentes técnicas e instrumentos que nos permitiesen la profundización en dichos temas antes de finalizar el curso.

Todas las fases han estado encadenadas $y$, aunque los resultados reflejados en este manuscrito corresponden en su mayoría a los datos recogidos en la tercera fase, estos no pueden comprenderse sin el carácter longitudinal del estudio y todos los pasos previos que se diseñaron y vivieron junto a las maestras, así como todo el conocimiento que ellas adquirieron durante los cursos anteriores. Además, aunque ya hemos reflejado el enfoque general de la investigación, para el estudio específico en torno a la evaluación que presentamos en este manuscrito el enfoque se delimitó al proceso de transformación de la evaluación de las maestras desde que comenzaron su participación en el PID-FPEIAP.

\section{Técnicas de recogida de datos}

Las técnicas y los instrumentos utilizados en la toma de datos se fueron creando y adaptando durante toda la investigación en función de las características y las necesidades de las participantes en cada momento. En esta forma de recoger los datos se buscó que la planificación de recogida de datos no condicionase la investigación, sino que la impulsase a través de su posibilidad de adaptación (Kincheloe, McLaren, Steinberg, \& Monzó, 2017). De esta forma la recogida de datos se realizó con diferentes técnicas e instrumentos durante la duración de la investigación, como se refleja en la Tabla 1. 
Tabla 1

Técnicas de recogida de datos utilizadas

\begin{tabular}{ll}
\hline Técnica & Total \\
\hline Entrevistas individuales & 15 \\
Grupo de discusión & 1 \\
Observaciones participantes & 34 \\
Cuestionarios cualitativos & 24 \\
Análisis documental & 4 \\
\hline
\end{tabular}

Para la exposición de los resultados en este artículo únicamente se han utilizado extractos de dos instrumentos: el grupo de discusión y la observación participante, al ser con los que se profundizó junto a las maestras en el tema de la evaluación.

Respecto al grupo de discusión, este se organizó al final de la tercera fase de la recogida de datos y tuvo como finalidad profundizar en algunos de los temas que habían surgido durante el Proyecto. Para ello se realizó un guion con los temas, se definieron unos objetivos y preguntas de investigación específicas en torno a ellos y, finalmente, se desarrollaron unas preguntas abiertas para los participantes. La finalidad de organizar así la toma de datos era la búsqueda de generar un debate entre los participantes en torno a una temática y no la contestación de una pregunta cerrada (Aarnio, Lindblom-Ylänne, Nieminen, \& Pyörälä, 2014). Se realizaron un total de nueve preguntas abiertas agrupadas en cinco temas. En la Tabla 2 se pueden observar las referidas a la evaluación.

Tabla 2

Guion de preguntas del grupo de discusión respecto al tema de la evaluación

\begin{tabular}{|c|c|c|}
\hline Objetivos & Preguntas de investigación & Preguntas para los participantes \\
\hline $\begin{array}{l}\text { - Analizar en qué grado se } \\
\text { aplican sistemas y buenas } \\
\text { prácticas de evaluación } \\
\text { formativa en el ejercicio } \\
\text { profesional de las maestras } \\
\text { y su relación con la } \\
\text { formación inicial recibida } \\
\text { - Analizar si la formación } \\
\text { permanente del PID-FPEIAP } \\
\text { ha ayudado a las docentes a } \\
\text { transformar sus prácticas de } \\
\text { evaluación }\end{array}$ & $\begin{array}{l}\text { ¿Cómo evalúan las } \\
\text { docentes a su alumnado? } \\
\text { ¿En qué medida la forma } \\
\text { de evaluar que utilizan las } \\
\text { docentes está influida por } \\
\text { la evaluación que vivieron } \\
\text { durante su formación } \\
\text { inicial? } \\
\text { ¿La participación de las } \\
\text { maestras en el PID-FPEIAP } \\
\text { ha modificado su forma de } \\
\text { evaluación? }\end{array}$ & $\begin{array}{l}\text { Otro de los temas que estamos } \\
\text { investigando es si en vuestra } \\
\text { práctica docente estáis llevando } \\
\text { a cabo sistemas y procesos de } \\
\text { evaluación formativa y, en caso } \\
\text { afirmativo, en qué medida la } \\
\text { forma de evaluar que utilizáis } \\
\text { está influida por la evaluación } \\
\text { que vivisteis durante vuestra } \\
\text { formación inicial. } \\
\text { Otro aspecto complementario } \\
\text { es si vuestra participación en } \\
\text { el PID-FPEIAP os ha ayudado a } \\
\text { modificar, trasformar o mejorar } \\
\text { la forma de evaluar a vuestro } \\
\text { alumnado. }\end{array}$ \\
\hline
\end{tabular}


En el grupo de discusión participaron seis maestras y un maestro del PID-FPEIAP. Esta decisión se tomó al ser siete participantes un número idóneo para procurar el debate y la interacción (Mena \& Méndez, 2009). Para la elección de la muestra de participantes se tuvo en cuenta la diversidad de estos en relación a los siguientes factores: (a) centro o centros educativos en los que habían dado clase los participantes durante su participación, buscando así una variedad de contextos en los que se desarrolló el PID-FPEIAP; (b) el género: al ser la mayor parte de las participantes mujeres, se procuró que esta proporción también se diese en el grupo de discusión; (c) los años de participación en el Proyecto: se seleccionaron participantes que accedieron en diferentes años, pero se seleccionaron únicamente docentes que llevasen en el Proyecto al menos un año y hubiesen tenido una participación activa; $y$ (d) factores personales y sociales que los investigadores consideramos clave para el desarrollo profundo de los temas a través del debate, tales como su formación externa, los años de experiencia o su capacidad de liderazgo.

Los datos extraídos en el grupo de discusión fueron expuestos y debatidos entre todos los participantes del PID-FPEIAP a través de unas jornadas internas del Proyecto en el año 2018. Los nuevos datos surgidos en dichas jornadas se recogieron en el diario de investigación.

El diario de investigación fue el instrumento utilizado para la recolección de las observaciones y reflexiones realizadas por el investigador. El diario se trata de una herramienta útil para la realización de procesos reflexivos críticos, pues permite ampliar el pensamiento acerca de los acontecimientos vividos (González-Calvo, 2013). El diario se utilizó para tomar datos en las reuniones mensuales, en las visitas a las aulas y colegios de las maestras participantes, las jornadas organizadas anualmente por el Proyecto, las conversaciones informales con las maestras en espacios neutros y otros eventos asociados al Proyecto, como la participación de las maestras en congresos o ponencias para narrar sus experiencias en el PID-FPEIAP.

\section{Análisis de datos}

Se ha realizado un análisis en profundidad de las voces e interacciones de las maestras con la finalidad de incidir en el objeto de estudio de esta investigación: el proceso de transformación de la evaluación de las maestras desde que comenzaron su participación en el PID-FPEIAP. El análisis de los datos se produjo de manera inductiva, la información ha provenido directamente de las voces de los actores principales de la investigación (Miron \& Mevorach, 2014), las cuales han guiado la construcción de las categorías y, posteriormente, las proposiciones teóricas del estudio (Hernández Carrera, 2014).

Se produjeron dos fases en el análisis de los datos: (1) una primera fase donde analizamos la información recogida de todas las técnicas e instrumentos utilizados durante las tres fases y la clasificamos en función del aporte al objeto de estudio, asegurándonos de obtener información detallada y una saturación de los datos; y (2) con los datos ya clasificados, los cruzamos para obtener temas coincidentes y generar así las categorías de análisis (Saldaña, 2009).

Las categorías de análisis a través de las cuáles se ha diseñado el estudio han sido: (a) la evaluación conocida y desarrollada por las maestras antes de su participación en el PIDFPEIAP: en esta categoría se incluyeron todos aquellos extractos en los que las maestras hacían referencia a cómo evaluaban antes de participar en el Proyecto, así como 
los conocimientos que poseían sobre la temática. Los datos reflejaron tres subtemas sobre los que se construyó el discurso en esta categoría: los conocimientos previos sobre evaluación, los aspectos qué evaluaban y cómo evaluaban; y (b) las concepciones de evaluación y su uso por parte de las maestras a raíz de su participación en el PID-FPEIAP, en esta categoría se analizaron las nuevas concepciones sobre evaluación que reflejaban los discursos de las maestras y sus nuevas prácticas de evaluación. Esta categoría también se construyó en torno a tres subtemas: las nuevas concepciones sobre evaluación, los aspectos qué evalúan y cómo evalúan.

Toda la categorización y codificación se llevó a cabo a través del software de análisis cualitativo de datos ATLAS.ti (2015).

\section{Análisis y resultados}

El análisis y los resultados se han construido en torno a dos variables: previo a la participación de las maestras en el PID-FPEIAP y posterior a la participación de las maestras en el PID-FPEIAP

\section{La evaluación conocida y desarrollada por las maestras antes de su participación en el PID-FPEIAP}

En esta categoría abordaremos los conocimientos previos que las maestras tenían sobre evaluación, así como los criterios que evaluaban y las técnicas e instrumentos que usaban antes de su participación en el Proyecto, tratando de profundizar en las ideas subyacentes, su formación y la relación entre todos estos factores.

Cuando las maestras comienzan en el PID-FPEIAP no concebían la evaluación como un medio de aprendizaje de su alumnado. Según afirmaban, la evaluación aprendida durante su formación inicial se basaba en la observación sistemática que el docente hace del aula, tal y como refleja la ley para la etapa de Educación Infantil (Real Decreto 1630/2006). Las maestras no hacían ningún tipo de alusión a otros agentes en la evaluación ni a prácticas de evaluación que fomentasen la participación del alumnado. Su bagaje académico y formativo les incitaba a entender la evaluación como un proceso externo al alumnado y a otros agentes educativos, y centrado exclusivamente en la observación y reflexión del docente.

Cuando salí de la carrera [...] la evaluación que te comentan en infantil es la observación sistemática, la teoría sobre la observación sistemática. (GD3, Junio de 2016).

Además de lo ya relatado, se destaca que las maestras decían haber aprendido sobre evaluación únicamente de manera teórica durante su formación académica. Las participantes reflejaban en sus discursos que su conocimiento sobre evaluación antes de participar en el PID-FPEIAP provenía de cuando, en su formación inicial, un profesor, o varios, les "comentaron" (en palabras de las docentes) el tipo de evaluación que se debe hacer en Educación Infantil. Las maestras relataban un aprendizaje sobre evaluación basado en una formación inicial técnica, donde los conocimientos habían sido desarrollados únicamente desde la teoría y las clases magistrales (Grundy, 1998), un enfoque incoherente para enseñar una evaluación participativa o democrática (Palacios \& López-Pastor, 2013). Por tanto, entender la evaluación como un proceso que le 
pertenece al docente no provenía solo del aprendizaje adquirido, sino también de la forma en la que lo habían desarrollado.

El aprender sobre evaluación desde un enfoque curricular técnico les supuso la pérdida de un compromiso personal, cultural y político con la educación (Luengo \& Saura, 2013). Este aspecto se vio claramente reflejado cuando las maestras dialogaron en el grupo de discusión acerca de los aspectos que solían observar en sus aulas y, por tanto, que evaluaban de su alumnado. Dichas observaciones estaban poco relacionadas con el aprendizaje individual, el aprendizaje social, la contextualización, los métodos o aspectos pedagógicos y, sin embargo, estaban centrados, principalmente, en el control y la eficacia. De esta forma, en el discurso de las maestras vuelve a vislumbrarse un uso de la evaluación sin tener en cuenta las individualidades del alumnado, sus momentos de desarrollo o sus circunstancias, otorgando siempre un carácter general y comparativo a la clase.

Antes yo observaba como muy a todos a la vez, todos con el libro, todos con la ficha. Y bueno, este que siempre acaba después y este que es el que acaba antes y poco más. (GD3, Junio de 2016).

Esta afirmación de las maestras se comprende como lógica si se tiene en cuenta la forma en la que habían aprendido sobre evaluación. La formación basada en clases magistrales teóricas no dotó a las maestras de las competencias necesarias para desarrollar su labor docente en el futuro, al no adquirir un pensamiento crítico (Barba, 2011); y, por ello, para llevar a cabo la evaluación se aferraron a sus vivencias, la mayoría de ellas relacionadas con una evaluación tradicional (López-Pastor, 2006). Así podemos comprobar cómo las participantes estaban continuando las prácticas de evaluación vividas, sin habérselas cuestionado, reproduciendo modelos tradicionales en los que el interés de la evaluación recaía en el resultado final (López-Pastor \& Palacios, 2012). Este aspecto vuelve a resaltarse con más énfasis cuando profundizamos en las técnicas e instrumentos que utilizaban para evaluar y el uso que les daban.

Las maestras antes de comenzar en el PID-FPEIAP asociaban el tema de la evaluación únicamente al uso de los boletines de evaluación finales que entregaban a los padres. Este instrumento se debe entregar a los padres al finalizar cada trimestre y es una parte del proceso de evaluación en Educación Infantil (Díaz \& Velasco, 2018). El problema radica en que las maestras únicamente hacían referencia a esta herramienta al referirse a la evaluación que realizaban.

Yo cuando empecé a trabajar, pues empiezas con [...] el boletín típico de "Conoce del 1 al 3, conseguido no conseguido, entiende el proceso" [...] y es cómo vas evaluando. (GD7, Junio de 2016).

Las docentes explican que el modelo de este instrumento que utilizaban se basaba en ítems cerrados y generales para todos los alumnos. Ellas debían ir rellenando con una " $X$ " cada categoría, en función del grado de desarrollo del alumnado respecto a ese ítem. A veces podían elegir entre varias opciones graduales entre el "conseguido" y el "no conseguido", mientras que otras veces solo podían seleccionar "sí" o "no".

Este modelo de boletín refleja un uso de la evaluación poco centrado en las individualidades de cada alumno. El propio instrumento condenaba la posibilidad de un uso participativo de la evaluación, al tener que rellenar unos ítems preestablecidos de aprendizaje y no graduales desde el comienzo iguales para todo el alumnado. 


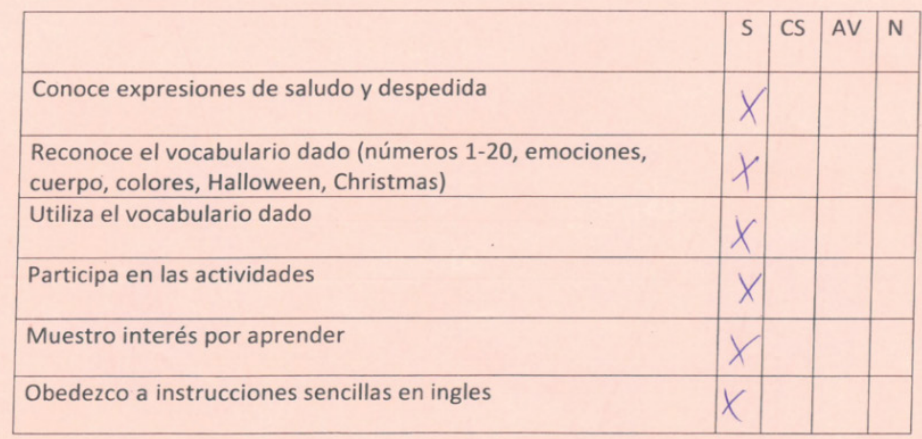

S: SIEMPRE CS: CASI SIEMPRE AV: AVECES N: NUNCA

Figura 1. Boletín de evaluación informativo solicitado a las maestras

Al indagar en el porqué del uso de este modelo de boletín, las maestras reflejaron que les era exigido por el propio centro o las administraciones territoriales.

A mí me pasa que me obligan a darlo. [refiriéndose al boletín] (GD4, junio de 2016)

Este hecho vuelve a relacionarse de forma fehaciente con la forma de aprender sobre evaluación en su formación inicial. El discurso de las maestras refleja la falta de un pensamiento crítico con el que cuestionarse las malas praxis, resultado de no haber sido participe en sus evaluaciones como estudiantes (Sivan, 2000), ni haber recibido una formación académica emancipadora (Imbernón, 2017).

Las maestras no se habían planteado la posibilidad de liberarse de quién les obligaba a realizar este modelo de boletín y asumían sus argumentos como ciertos. Sin embargo, más adelante en el Proyecto, cuando volvió a surgir el tema del boletín de evaluación, las propias docentes comprobaron que los argumentos expresados por quienes les obligaban a realizar el modelo general de boletín no eran ciertas, pues no existe un modelo estandarizado obligatorio de evaluación para Educación Infantil (Real Decreto 1630/2006).

En la última reunión quedamos en que para hoy íbamos a haber mirado en la ley sobre el tipo de informe de evaluación que se pide en Educación Infantil. Resulta que, tras analizar el documento entre todos, no existe ni el más mínimo indicio de que haya un informe estandarizado que las maestras tengan que cumplir. (Diario, mayo de 2018)

El uso del principal instrumento de evaluación desarrollado por las maestras estaba basado en una relación de poder. La falta de una participación en su evaluación cuando eran estudiantes y una formación inicial alejada de posturas emancipadoras, hizo que las maestras llegasen a asumir prácticas de evaluación tradicionales impuestas por agentes externos, cuyas argumentaciones para obligarlas a su uso se apoyaban en preceptos no reflejados por la ley.

Los resultados de esta categoría muestran cómo el discurso de las maestras antes de participar en el PID-FPEIAP se asociaba a una evaluación tradicional y una falta de 
crítica en su formación académica. Sus conocimientos, sus procesos de observación y sus instrumentos solían tener fines de calificación, de carácter general para toda el aula y poco participativos con otros agentes educativos. Además se basaban en sus vivencias como alumnas o en imposiciones externas. Estos hechos se relacionan con haber conocido cómo se realizaban las evaluaciones de forma teórica en la formación inicial, no haber vivenciado una evaluación participativa como estudiantes y no haber trabajado una reflexión crítica.

\section{Las concepciones de evaluación y su uso por parte de las maestras a raíz de su participación en el PID-FPEIAP}

Cuando las maestras comienzan en el Proyecto este les proporciona un espacio para la reflexión y la profundización de sus concepciones educativas. La formación que extraen las maestras en el PID-FPEIAP, basada en la promoción de la educación inclusiva, comenzó a traspasar las primeras prácticas que llevaron a cabo y se instauró en su día a día, en sus prácticas escolares, en sus relaciones, en sus observaciones y también en sus formas de evaluar.

En el nuevo discurso de las maestras se podía observar una transformación del mismo al referirse a la evaluación. Ya no hablaban únicamente de la evaluación como un aspecto final, destinado exclusivamente a obtener información para la calificación y basado en su propio punto de vista a través de la observación sistemática; sino que consideraban que, a raíz de su participación en el Proyecto, habían comenzado a comprender la evaluación como un proceso participativo del alumnado, con el cual hacerle consciente de su aprendizaje y mejorarlo a través del uso de feedback en el momento (Brown \& Pickford, 2006; Dann, 2014).

Al principio no lo hacía, pero luego vi que era necesario hacer una pequeña asamblea [...] y hablar entre todos: "¿y a qué hemos jugado?, ¿y que hemos hecho?, ¿y a dónde has ido?" y entre ellos también, es un poco la coevaluación. (GD3, junio de 2016)

En sus nuevos relatos sobre evaluación, las maestras afirmaban haber olvidado aquellas propuestas de evaluación iniciales, en las que todo partía únicamente de su observación sistemática. Ahora reflejaban la utilización de otras formas de evaluación más participativas, como la autoevaluación o la evaluación entre pares.

Hemos introducido la autoevaluación de los alumnos [...] yo creo que, principalmente, les hemos hecho participes. Y la coevaluación. Porque siempre era la evaluación nuestra. (GD6, junio de 2016).

Los nuevos discursos de las maestras mostraban su relación con el tipo de aprendizaje fomentado desde el PID-FPEIAP a través de procesos de indagación autoreflexivos. Las docentes hacían referencia a cómo la reflexión les hizo replantearse un cambio en su evaluación y cómo se dieron cuenta de que no debía ser un proceso únicamente suyo. La reflexión crítica les permitió transformar sus prácticas de evaluación al cuestionárselas y dirigirlas en la búsqueda de una educación para la democracia en sus aulas, a través de la cual los niños se fuesen implicando progresivamente en su aprendizaje y en la que todas las directrices no se imponían al criterio de los adultos (Meirieu, 2004). 
Estas nuevas perspectivas de evaluación y los modelos utilizados también cambiaron sus observaciones en las aulas. Las maestras comenzaron a hablar de comportamiento, silencio o control cuando se refieren a evaluar, y lo hacían en términos asociados a la inclusión o la democratización, como el diálogo, las interacciones, las relaciones o el aprendizaje entre iguales.

Ya como que tiene que ser todo, todo, inclusivo. Que en todos los sitios los alumnos aprendan a ayudarse, aprender a respetarse, a colaborar. (GD3, junio de 2016).

En este nuevo discurso las maestras evidencian cómo prestan atención y observan, principalmente, lo que para ellas ahora es importante fomentar en sus aulas y, por tanto, evaluar. Ya no se basan en aspectos fácilmente calificables y que apenas aportaban aprendizaje, sino que centran sus observaciones en conocer al alumnado, en sus relaciones o en sus formas de aprender. Por tanto, se denota, una transformación de su pensamiento como docentes. La conciencia pedagógica crítica que han adquirido gracias al análisis de sus realidades escolares les ha permitido dotar de valor a aspectos tan importantes como la inclusión o el aprendizaje desde la colaboración.

En cuanto a los instrumentos utilizados, estos también han sido transformados acorde al nuevo pensamiento educativo de las maestras.

Yo a la hora de evaluar [...] tengo una manera diferente de dónde registrar [...], que antes no lo tenía de esa manera puesto. (GD7, junio de 2016).

Respecto al boletín de evaluación final que analizábamos anteriormente, las maestras han decidido transformarlo por otro cualitativo, redactado e individualizado, basado en la autoevaluación del alumnado (Barba-Martín, 2019). Este cambio fue muy cuestionado en el contexto de las maestras, debido al desconocimiento o la posibilidad de que se tambaleasen algunas posiciones cómodas de poder (Freire, 1968/2007). Sin embargo, ellas siguieron adelante y buscaron soluciones para implantar este nuevo instrumento, lo cual refleja la adquisición de una pedagogía crítica por parte de las maestras, capaz de romper con las relaciones de poder (Zeichner, 2010).

El modelo formativo emancipador del PID-FPEIAP fue el aspecto clave para romper con los modelos de evaluación obligados. El proceso de transformación hacia el nuevo modelo de boletín se inició cuando las maestras comenzaron a analizar todo lo que acontecía su práctica, tratando de sacarle el mayor partido posible al aprendizaje de su alumnado y hacerle más participativo. Así el relato de las maestras nos muestra cómo llegaron a un proceso de reflexión que les hizo comprender el escaso sentido que tenía un boletín de ítems cerrados y estandarizado, cuando ellas querían promocionar en su aula valores de inclusión, equidad o relaciones igualitarias, las cuales estaban en línea con una evaluación democrática (McDonald, 1976).

Nosotros nos hemos dado cuenta que no sirve eso, estamos en la reflexión de "Eso lo tenemos que cambiar" [...] Oye, estos boletines no tienen ningún sentido. (GD6, junio de 2016).

Para las maestras la evaluación ya había dejado de tener un carácter sumativo y final, lo que supuso la necesidad de utilizar otros instrumentos. El diario del profesor adquirió un valor importante para reflexionar y poder realizar una evaluación detallada de cada alumno en el momento de su aprendizaje, así como para recoger las autoevaluaciones del alumnado y las evaluaciones compartidas. Además, al haber ampliado aún 
más sus miras y comprendido cómo la mejora del aprendizaje se situaba en muchos aspectos, las docentes afirmaban que este instrumentos también les estaba sirviendo para evaluarse a sí mismas y sus prácticas.

Ahora es que tengo la libreta aquí metida, y constantemente para evaluar a los niños y para evaluar tu propia práctica. Y yo a raíz del PID-FPEIAP evalúo más en el momento, y tomo notas y a apuntar, también es algo que he seguido haciendo el año pasado y este. (GD3, junio de 2016)

Esta utilización del diario del profesor se acerca a los fines formativos con los que la pedagogía crítica lo une. Las maestras encontraron en este instrumento un uso para registrar constantemente acontecimientos de sus clases y su alumnado, con el fin de ir evaluándolo para mejorarlos constantemente. Sin embargo, también descubrieron en el diario un uso formativo, el cual les permitió desarrollarse a través de procesos de reflexión-sobre-la-acción y un cuidadoso análisis de los presupuestos, ideas y conocimientos en los cuales se basaban sus prácticas (Perrenoud, 2007; Tagle, 2011).

La reflexión crítica subyacente a todas estas nuevas concepciones y usos de la evaluación ha permitido a las maestras asentar un pensamiento educativo y un modelo pedagógico críticos, consistentes con el fomento de una evaluación democrática (Fernández-Balboa, 2007; Lorente \& Kirk, 2014). La formación del PID-FPEIAP, basada en la investigación autorreflexiva y la educación inclusiva, les permitió romper las barreras creadas por las relaciones de poder que vivían hasta el momento, haciéndoles comprender que por encima de ellas debe estar la democratización de sus aulas, sus escuelas y sus prácticas de evaluación, donde todos los agentes educativos sean partícipes y estén informados de todo lo que acontece.

Yo una de las cosas que me ha aportado más el Proyecto es, sobre todo, la importancia de compartir, pero también de dar a conocer a los padres, de que ellos realmente sepan lo que están haciendo. [refiriéndose a los hijos e hijas] (GD1, junio de 2016).

\section{Discusión y conclusiones}

El análisis del proceso de transformación de la evaluación por parte de las maestras del PID-FPEIAP y su relación con los modelos formativos vividos, objetivo de la investigación, muestra cómo la formación docente basada en la adquisición de un pensamiento y un enfoque pedagógico crítico es importante para la ruptura de modelos tradicionales de evaluación, al ser capaz de desarrollar en el profesorado una preocupación por las individualidades del alumnado, el fomento de la participación y una educación para la democracia.

Estudios previos en el marco del PID-FPEIAP (Barba-Martín, 2019) muestran cómo la participación de las maestras en el Proyecto consiguió incidir en la construcción de una identidad docente crítica. Al ser una formación basada en la reflexión y asociada a la promoción de la educación inclusiva, contribuyó a una transformación del pensamiento docente, que influyó en la consolidación de nuevos conocimientos en las maestras, una transformación de su lenguaje, una preocupación por fomentar la democracia y la adquisición de un enfoque pedagógico crítico. Todo ello lo hemos visto reflejado en este estudio a través de la evaluación.

Los resultados muestran cómo en las maestras el modelo formativo técnico vivido se relaciona con la imposibilidad de poner en práctica una evaluación democrática. Por 
un lado, los datos extraídos en relación a antes de la participación de las maestras en el PID-FPEIAP evidencian una relación entre el modelo formativo técnico desarrollado durante su formación académica y el uso de prácticas de evaluación tradicionales cuando fueron maestras. El aprendizaje sobre evaluación desde un enfoque curricular basado en clases magistrales teóricas mostró una correspondencia con una concepción reducida de la evaluación por parte de las maestras y un uso despreocupado por la participación o la individualidad del alumnado. Estos aspectos fueron consecuencia de ser este un modelo curricular orientado a la reproducción y no a la reflexión o la vivencia. Estos resultados son coherentes con las investigaciones de Gargallo, Sánchez, Ros y Ferreras (2010) o Palacios y López-Pastor (2013), quienes defienden como los procesos de enseñanza-aprendizaje utilizados por el profesorado universitario tienen una relación directa con la evaluación que desarrolla y vivencia su alumnado.

Por otro lado, el relato de las docentes a raíz de su participación en el PID-FPEIAP muestra un concepto y uso de la evaluación participativa y democrática, relacionado con la formación crítica y reflexiva del Proyecto. El nuevo discurso de las maestras se ve impregnado de alusiones a nuevos conceptos participativos sobre evaluación, como la autoevaluación o la evaluación entre pares, pero también de reflexiones sobre los beneficios que suponen estos modelos en el desarrollo del aprendizaje de su alumnado y la promoción de una democracia en sus aulas. Los resultados muestran cómo estos nuevos conocimientos y usos de la evaluación se asentaron en las maestras a través del cuestionamiento de todas las ideas que subyacían a sus antiguas prácticas de evaluación. De esta forma el avance hacia los nuevos modelos de evaluación se produjo desde la profundización crítica en las relaciones, la ética y las injusticias de los modelos tradicionales. Lo cual se relaciona con pasos fundamentales para desarrollar verdaderos modelos de evaluación democráticos (Fernández-Balboa, 2007) o de justicia social (Murillo \& Hidalgo, 2015; Hidalgo \& Murillo, 2016).

En este sentido, se refleja la necesidad de desarrollar un pensamiento y enfoque pedagógico crítico durante la formación inicial del profesorado para poner en marcha una evaluación democrática. El primer paso debe ser la adquisición de un pensamiento crítico, que permita a los docentes cuestionarse los conocimientos adquiridos y el cual se puede fomentar desde la vivencia como estudiantes de una evaluación participativa (Fitzpatrick, 2006). Sin embargo, para lograr el asentamiento de una evaluación democrática, los resultados de la investigación demuestran que la formación inicial debe avanzar más, hacia el desarrollo de un enfoque pedagógico crítico en sus estudiantes, con el cual puedan cuestionarse todos los factores injustos intrínsecos a las prácticas de evaluación. Para lo cual es necesario eliminar los modelos curriculares técnicos y desarrollar los conocimientos a través de modelos emancipadores (González-Calvo \& Barba, 2014), formando así docentes comprometidos ética, social y políticamente, capaces de cuestionarse, desde estos tres aspectos, los valores y las relaciones subyacentes a todos sus métodos y prácticas, incluidos los de evaluación, para romper con aquellos injustos (Cochran-Smith, 2009; McLaren, 2005).

Siguiendo esta línea, el relato refleja que las características de la formación emancipadora del PID-FPEIAP fueron capaces de incidir en la ruptura con las relaciones de poder ocultas en algunas prácticas e instrumentos de evaluación tradicionales. Autores como Hamodi, López-Pastor y López-Pastor (2017) exponen que la aplicación de procesos e instrumentos de evaluación formativa y compartida durante la formación inicial del profesorado puede llegar a reflejarse en la futura labor docente de los estudiantes, haciendo que no reproduzcan los modelos de evaluación tradicionales vividos anteriormente y sí los formativos. Sin embargo, a través de los resultados del 
estudio, podemos ampliar esta conclusión a que el enfoque académico desde el que lo vivencien también es importante, ya que sin una capacidad de emancipación el profesorado seguirá asumiendo prácticas de evaluación tradicionales impuestas. El relato de las maestras señala cómo el instrumento que utilizaban para evaluar a su alumnado en Educación Infantil, antes de participar en el PID-FPEIAP, les venía obligado por sus centros escolares, sin ninguna base legal. Los resultados muestran cómo la adquisición de un enfoque pedagógico crítico, alcanzado a través de procesos de investigación autorreflexivos, es lo que permitió a las maestras replantearse la validez del instrumento, la veracidad de los argumentos y buscar propuestas alternativas. Esta situación concuerda con los planteamientos de autores referentes de la pedagogía crítica (Cochran-Smith, 2009; Freire, 1968/2007; McLaren \& Giroux, 1990; Zeichner, 2016), al considerar que este enfoque pedagógico es capaz de sobreponerse a las relaciones de poder existentes en las prácticas tradicionales. Por tanto, los datos vuelven a apoyar la necesidad de una formación inicial basada en el modelo emancipador, al ser sus características realmente influyentes para romper con prácticas y concepciones de evaluación tradicionales impuestas de forma ilícita desde las instituciones o las administraciones educativas.

Como conclusión final, el estudio nos ha mostrado la importancia que tiene el modelo curricular vivido en la formación inicial del profesorado para asentar una evaluación democrática en las aulas de Educación Infantil. Esta práctica de evaluación requiere de una formación académica capaz de conseguir que como estudiantes, y, posteriormente, como profesorado, sean capaces de cuestionarse los valores e ideales políticos, sociales y culturales subyacentes a las prácticas de evaluación tradicionales, principalmente, en su relación con el alumnado más desfavorecido. Por tanto, resulta necesaria la vivencia de una evaluación democrática durante la formación inicial del profesorado desde un modelo curricular emancipador, ya que de esta manera los futuros docentes conseguirán desarrollar unos conocimientos y un enfoque pedagógico crítico, siendo esta unión capaz de romper con los modelos tradicionales de evaluación.

Para finalizar, consideramos necesario comprender las conclusiones del estudio dentro de su contexto, ya que el estudio se encuentra restringido por este factor. En este sentido, sería positivo realizar una experiencia semejante en otros contextos, que permitiese una comparación de los resultados en cuanto a la relación entre el desarrollo de un enfoque pedagógico crítico y la ruptura con los modelos tradicionales de evaluación. Además, los resultados del estudio también nos animan a seguir avanzando en su profundización. Así, consideramos que pueden ser líneas interesantes de desarrollo el buscar las sinergias entre la promoción de una educación inclusiva y las bases de la evaluación compartida y democrática; o el análisis de experiencias que aúnen un enfoque pedagógico crítico y la vivencia de la evaluación compartida durante la asignatura de Prácticum.

\section{Referencias bibliográficas}

Aarnio, M., Lindblom-Ylänne, S. Nieminen, J., \& Pyörälä, E. (2014). How Do Tutors Intervene When Conflicts on Knowledge Arise in Tutorial Groups? Advances in Health Sciences Education, 19(3), 329-345. Doi: https://doi.org/10.1007/s10459013-9473-5 
Andrade, H., \& Du, Y. (2007). Student responses to criteria-referenced self-assessment. Assessment \& Evaluation in Higher Education, 32(2), 159-181. Doi: https:// doi.org/10.1080/02602930600801928

ATLAS.ti (versión 7.5.7) [software de análisis cualitativo] (2015). Berlín: GmbH

Barba, J. J. (2011). El desarrollo profesional de un maestro novel en la escuela rural desde una perspectiva crítica. (Tesis doctoral). Universidad de Valladolid, Segovia. Recuperado de http://uvadoc.uva.es/handle/10324/822

Barba-Martín, R. A. (2019). La investigación-acción participativa desde la mirada de las maestras participantes en un proceso de formación permanente del profesorado sobre educación inclusiva. (Tesis doctoral). Universidad de Valladolid, Segovia. Recuperado de http://uvadoc.uva.es/handle/10324/37920

Beackley, A. (2000). Adrift without a life belt: Reflective self-assessment in a post-modern age. Teaching in Higher Education, 5(4), 405-418. Doi: https://doi. org/10.1080/713699179

Boud, D., \& Falchikov, N. (Eds.). (2007). Rethinking assessment in higher education: Learning for the longer term. New York, US: Routledge.

Brown, S., \& Pickforf, R. (2006). Assessing skill and practice. London: Routledge

Cochran-Smith, M. (2009). Toward A Theory Of Teacher Education For Social Justice. En M, Fullam, A. Hargreaves, D. Hopkins, \& A. Lieberman, (Eds), The International Handbook of Educational Change (pp. 916-951). Nueva York: Springer Publishing

Dann, R. (2014). Assessment as learning: blurring the boundaries of assessment and learning for theory, policy and practice. Assessment in Education: Principles, Policy \& Practice, 21(2), 149-166. Doi: https://doi.org/10.1080/0969594X.2014.898128

Denzin, N. K., \& Lincoln, Y. S. (Coords.) (2012). Manual de investigación cualitativa. Barcelona: Gedisa.

Díaz, E. C., \& Velasco, A. J. (2018). Concepciones docentes acerca de la evaluación y el boletín informativo en educación inicial. Pensamiento Educativo. Revista de Investigación Educacional Latinoamericana, 55(1), 1-17. Doi: https://doi.org/10.7764/ PEL.55.1.2018.8

Dochy, F., Segers, M., \& Sluijsmans, D. (1999). The use of self, peer and co-assessment in higher education: A review. Studies in Higher Education, 24(3), 331-350. Doi: https://doi.org/10.1080/03075079912331379935

Fernández-Balboa, J. M. (2005). La autoevaluación como práctica promotora de la democracia y la dignidad. En A. Sicilia, \& J. M. Fernández-Balboa (Coords.), La otra cara de la educación física: la educación física desde una perspectiva crítica (pp. 127158). Barcelona: Inde.

Fernández-Balboa, J. M. (2007). Dignity and democracy in the college classroom: The practice of self-evaluation. En R.A. Goldstein (Ed.), Useful Theory: Making Critial Eduation Practical (pp. 105-128). Nueva York: Peter Lang Publishing.

Fitzpatrick, J. (2006). An evaluative case study of the dilemmas experienced in designing a self-assessment strategy for community nursing students. Assessment \& Evaluation in Higher Education, 31(1), 37-53. Doi: https://doi. org/10.1080/02602930500262361

Freire, P. (1968/2007). Pedagogía del oprimido (16a ed.). Madrid: Siglo XXI.

García Herránz, S., \& López-Pastor, V. M. (2015). Evaluación Formativa y Compartida en Educación Infantil. Revisión de una Experiencia Didáctica. Qualitative Research in Education, 4(3), 269-298. Doi: http://dx.doi.org/10.17583/qre.2015.1269 
Gargallo, B., Sánchez, F. J., Ros, C., \& Ferreras, A. (2010). Estilos docentes de los profesores universitarios: la percepción de los alumnos de los buenos profesores. Revista Iberoamericana de Educación, 51(4), 1681-5653. Doi: https://doi.org/10.35362/ rie5141826

Giroux, H. A. (2006). La escuela y la lucha por la ciudadanía. Pedagogía critica de la época moderna. México: Siglo XXI editores.

González-Calvo, G. (2013). Evolución de la identidad profesional de un docente novel de Educación Física: análisis a partir de la reflexión de sus experiencias personales y de su propia práctica. (Tesis Doctoral). Universidad de Valladolid, Valladolid. Recuperado de http://uvadoc.uva.es/handle/10324/2817

González-Calvo, G., \& Barba, J. J. (2014). Formación permanente y desarrollo de la identidad reflexiva del profesorado desde las perspectivas grupal e individual. Profesorado. Revista de Currículum y Formación del Profesorado, 18(1), 397-412. Recuperado de https://recyt.fecyt.es/index.php/profesorado/article/view/41093

Greenwood, D. J., \& Levin, M. (2007). Introduction to Action Research. Social Research for Social Change (2nd ed). Thousand Oaks, California: Sage Publications, Inc.

Grundy, S. (1998). Producto o praxis del currículum. Madrid: Morata.

Guzmán-Valenzuela, C. (2014). Polos epistemológicos, uso y construcción de teoría en investigación cualitativa en educación. Magis, Revista Internacional de Investigación en Educación, 7(14), 15-28. Doi: https://doi.org/10.11144/Javeriana.M7-14. PEUC

Hamodi, C., López-Pastor, V. M., \& López, A. T. (2017). If I experience formative assessment whilst at University will I put it into practice later as a teacher? Formative and shared assessment in Initial Teacher Education (ITE). European Journal of Teacher Education, 40(2), 171-190. Doi: https://doi.org/10.1080/02619768.2017.128 1909

Hernández Carrera, R. M. (2014). La investigación cualitativa a través de entrevistas. Su análisis mediante la teoría fundamentada. Cuestiones Pedagógicas, 23, 187-210. Doi: http://dx.doi.org/10.12795/CP

Herrero-Nivela, M. L., Escolano-Pérez, E., Anguera, M. T., \& Acero-Ferrero, M. (2014). Evaluación mediante observación de la motricidad en niños de Educación Infantil. International Journal of Developmental and Educational Psychology, 5(1), 469475. Doi: https://doi.org/10.17060/ijodaep.2014.n1.v5.708

Hidalgo, N., \& Murillo, F. (2016). Evaluación de estudiantes para la justicia social. Propuesta de un modelo. Revista Internacional de Educación para la Justicia Social, 5(2), 159-179. Doi: https://doi.org/10.15366/riejs2016.5.2.008

Hortigüela, D., \& Pérez-Pueyo, A. (2016). La evaluación entre iguales como herramienta para la mejora de la práctica docente. Opción: Revista de Ciencias Humanas y Sociales, 7, 865-879. Recuperado de https://produccioncientificaluz.org/index. php/opcion/article/view/21509

Huang, Y. M., Hsu, C. C., Su, Y. N., \& Liu, C. J. (2014). Empowering Classroom Observation with an E-Book Reading Behavior Monitoring System Using Sensing Technologies. Interacting with Computers, 26(4), 372-387. Doi: https://doi.org/10.1093/ iwc/iwu012

Imbernón, F. (2017). Ser docente en una sociedad compleja: La difícil tarea de enseñar. Barcelona: Graó. 
Kemmis, S. (2011). A Self-Reflective Practitioner and a New Definition of Critical Participatory Action Research. En N. Mockler \& J. Sachs (eds), Rethinking Educational Practice Through Reflexive Inquiry (pp. 11-29). Dordrecht: Springer.

Kemmis, S., McTaggart, R., \& Nixon, R. (2015). Critical theory and critical participatory action research. En $\mathrm{H}$. Bradbury (Ed.), The SAGE Handbook of action research (pp. 453-464). City Road, London: SAGE.

Kennedy, A., \& Allan, J. (2009). The assessor and the assessed: Learning from students' reflections on peer evaluation. Journal of Teacher Education and Teachers' Work, 1(1), 4-17. Recuperado de https://strathprints.strath.ac.uk/28523/

Kincheloe, J. L. (2012). Teachers as researches. Qualitative inquiry as a path to em powerment ( $4^{\mathrm{a}}$ ed.). Wiltshire: Routledge.

Kincheloe, J. L., McLaren, P., Steinberg, S., \& Monzó, L. (2017). Critical pedagogy and qualitative research: Advancing the bricolage. En N. K. Denzin, \& Y. S. Lincoln (Eds.), The SAGE Handbook of Qualitative Research (5th ed.) (pp. 235-260). Thousand Oaks, CA: Sage.

Lopez-Benavente, A., Urena-Ortin, N., \& Lopez, F. (2019). Formative and shared assessment for the inclusion of active breaks in kindergarten. Journal of Sport and Health Research, 11(1), 143-154. Recuperado de http://www.journalshr.com/index.php/ issues/2019

López-Pastor, V. M. (2006). La evaluación en educación física. Revisión de los modelos tradicionales y planteamiento de una alternativa: La evaluación formativa y compartida. Buenos Aires: Miño y Dávila.

López-Pastor, V. M., \& Palacios, A. (2012). Percepción de los futuros docentes sobre los sistemas de evaluación de sus aprendizajes. Revista Teoría de la Educación: Educación y Cultura en la Sociedad de la Información, 13, 317-340. Doi: http://dx.doi. org/10.14201/eks.9143

López-Pastor, V. M., \& Pérez-Pueyo, A. (coords.) (2017). Evaluación formativa y compartida en educación: experiencias en todas las etapas educativas. León: Universidad de León.

López-Pastor, V. M., Castejón, J., Sicilia-Camacho, A., Navarro-Adelantado, V., \& Webb, G. (2011). The process of creating a cross-university network for formative and shared assessment in higher education in Spain and its potential applications. Innovations in Education and Teaching International, 48(1), 79-90. Doi: https://doi. org/10.1080/14703297.2010.543768

Lorente, E., \& Kirk, D. (2013). Making the case for democratic assessment practices within a critical pedagogy of physical education teacher education. European Physical Education Review, 20(1), 104-119. Doi: https://doi.org/10.1177/1356336X13496004

Lorente, E., \& Kirk, D. (2014). Alternative democratic assessment in PETE: an action-research study exploring risks, challenges and solutions. Sport, Education and Society, 18(1), 77-96. Doi: https://doi.org/10.1080/13573322.2012.713859

Luengo, J., \& Saura, G. (2013). La performatividad en la educación. La construcción del nuevo docente y el nuevo gestor performativo. Revista Iberoamericana sobre Calidad, Eficacia y Cambio en Educación, 11(3), 139-153. Recuperado de https:// revistas.uam.es/index.php/reice/article/view/2909

Manrique, J. M., López-Pastor, V. M., Monjas, R., \& Real, F., (2010). El potencial de los proyectos de aprendizaje tutorado y los sistemas de evaluación formativa en la mejora de la autonomía del alumnado. Una experiencia interdisciplinar. Revista 
Española de Educación Física y Deportes, 14(V), 39-57. Recuperado de http://www. reefd.es/index.php/reefd/article/view/285

McDonald, B. (1976). Evaluation and the control of education. En D. Tawney (Ed.), Curriculum evaluation today: Trends and implications. (pp. 125-134). London: McMillan Education

McLaren, P. (2005). La vida en las escuelas. Una introducción a la pedagogía crítica en los fundamentos de la educación ( $4^{\mathrm{a}}$ ed.). Buenos Aires: Siglo XXI.

McLaren, P., \& Giroux, H. A. (1990). La educación del profesorado como espacio contrapúblico: Apuntes de una redefinición. En T. S. Popkewitz (Coord.), Formación de profesorado. Tradición. Teoría. Práctica (pp. 244-271). Valencia: Universitat de Valencia.

Meirieu, P. (2004). En la escuela hoy. Barcelona: Octaedro.

Mena, A. M., \& Méndez, J. M. (2009). La técnica de grupo de discusión en la investigación cualitativa. Aportaciones para el análisis de los procesos de interacción. Revista Iberoamericana De Educación, 49(3), 1-7. Doi: https://doi.org/10.35362/ rie4932094

Miron, M., \& Mevorach, M. (2014). The "Good Professor" as Perceived by Experienced Teachers Who Are Graduate Students. Journal of Education and Training Studies, 2(3), 82-87. Doi: https://doi.org/10.11114/jets.v2i3.411

Murillo., F., \& Hidalgo, N. (2015). Enfoques fundamentales de evaluación de estudiantes para la justicia social. Revista Iberoamericana de Evaluación Educativa, 8(1), 4361. Recuperado de https://revistas.uam.es/riee/article/view/2975

Palacios, A., \& López-Pastor, V. M. (2013). Haz lo que yo digo pero no lo que yo hago: sistemas de evaluación del alumnado en la formación inicial del profesorado. Revista de Educación, 361, 279-305. Doi: https://doi.org/10.4438/1988-592XRE-2011-361-143

Páramo, M. B., \& Castro, M. (2017). Prácticas de evaluación en Educación Infantil un proyecto globalizado y en comunidad. Revista de Estudios e Investigación en Psicología y Educación, 10, 47-52. Doi: https://doi.org/10.17979/reipe.2017.0.10.2573

Perrenoud, P. (2007). Desarrollar la práctica reflexiva en el oficio de enseñar (3a ed.). México: Graó.

Real Decreto/1630/2006, de 29 de diciembre, por el que se establecen las enseñanzas mínimas del segundo ciclo de Educación infantil. Boletín Oficial del Estado, 4, Ministerio de Educación y Ciencia, Madrid, España, 04 de enero de 2007.

Rodríguez, G., Ibarra, M. S., \& Gómez, M. A., (2011). e-Autoevaluación en la universidad: un reto para profesores y estudiantes. Revista de Educación, 356, 401-430. Doi: https://doi.org/10-4438/1988-592X-RE-2010-356-045

Saldaña, J. (2009). The Coding Manual for Qualitative Researchers. Thousand Oaks, CA: Sage.

Santos Guerra, M. A. (1993). La evaluación, un proceso de diálogo, comprensión y mejora. Ed. Aljibe: Archidona

Santos Guerra, M. A. (2003). Dime cómo evalúas y te diré qué tipo de profesional y de persona eres. Revista Enfoques Educacionales, 5(1), 01-15. Recuperado de https:// enfoqueseducacionales.uchile.cl/index.php/REE/article/view/47513

Sivan, A. (2000). The implementation of peer assessment: an action research approach. Assessment in Education, 7(2), 193-213. Doi: https://doi.org/10.1080/713613328 
Tagle, T. (2011) El enfoque reflexivo en la formación docente. Calidad en la Educación, 34, 203-215. Doi: http://dx.doi.org/10.31619/caledu.n34.136

Tan, K. (2008). Qualitatively different ways of experiencing student self-assessment. Higher Education Research and Development, 27(1), 15-29. Doi: https://doi. org/10.1080/07294360701658708

Zeichner, K. M. (2010). Nuevas epistemologías en formación del profesorado. Revista Interuniversitaria de Formación del Profesorado, 68(24,2), 123-149. Recuperado de https://dialnet.unirioja.es/servlet/articulo?codigo=3276054

Zeichner, K. M. (2016). Promoviendo la justicia social y la democracia en la formación del profesorado preparación del profesorado 1.0, 2.0 y 3.0. Revista internacional de educación para la justicia social (RIEJS), 5(2), 15-23. Doi: https://doi. org/10.15366/riejs2016.5.2.001 